\title{
The predatory behaviour of Monopelopia tenuicalcar (Kieffer, 1918) larvae in a laboratory experiment
}

\author{
Vít SYROVÁTKA* \\ Department of Botany and Zoology, Faculty of Science, Masaryk University, Kotlářská 2, 61137 Brno, Czech Republic \\ *Corresponding author: syrovat@sci.muni.cz
}

\begin{abstract}
Larvae of the subfamily Tanypodinae are in general regarded as predators. Actual predation has been observed directly in only a few Tanypodinae species, but their behaviour and mouthpart morphology suggest that all Tanypodinae ingest food in the same way and thus are all predators. This view is reflected in most autecological databases. There remains uncertainty for some species, most notably for Monopelopia tenuicalcar (Kieffer, 1918). The uncertainty stems from the lack of direct observations, while gut content analysis points to non-animal food sources. A laboratory experiment was carried out in which larvae of Corynoneura sp. were offered to M. tenuicalcar in a set of Petri dishes. All predator and prey larvae were collected from the same locality, where they were the most abundant members of early spring littoral community. $M$. tenuicalcar showed clear predatory behaviour. In most cases (84 out of 86) the predator larva pierced the larva of Corynoneura and sucked its inner body content instead of engulfing it. Only in two cases did the predator engulf the whole victim. In all cases the seizing and processing of the prey was the same, with the ingestion of the food carried out by strong sucking. Obviously, if the chitinous structures of the prey fit the mouth opening of the predator, it was engulfed completely, otherwise the prey larva's internal contents were just sucked out. A video of the exceptional case of engulfing the whole prey is available at https://youtu.be/o6-8dA1XDy0. The strategy of sucking out prey may explain why body remnants like head capsules or claws of prey have not been found in the guts of Monopelopia larvae. Instead, the guts of Monopelopia larvae that pierced Corynoneura were green as they were full of algae "stolen" from the intestines of the grazing victims. Piercing and sucking out prey by Tanypodinae may be more common than has been expected before. Due to this, gut content analysis seems to be unreliable for the determination of feeding strategy in Tanypodinae.
\end{abstract}

Key words: Monopelopia tenuicalcar; Corynoneura sp.; predation; autecology; Tanypodinae; feeding; food webs; Sphagnum bog.

Received: February 2018. Accepted: May 2018.

This paper was presented at the $20^{\text {th }}$ International Symposium on Chironomidae, Trento, Italy, 2-8 July 2017.

Session: Autecology and Physiology.

\section{INTRODUCTION}

Monopelopia tenuicalcar (Kieffer, 1918) is the only representative of the genus Monopelopia known from Europe. With a body length up to $6 \mathrm{~mm}$ (Andersen et al.,2013) M. tenuicalcar is one of the smallest species within the subfamily Tanypodinae (the third largest subfamily of Chironomidae with 575 species recognized worldwide, Ashe and O'Connor 2009). Larvae of Tanypodinae differ markedly from the remaining Chironomidae in several characters, most importantly by the presence of ligula - a chitinous structure developed on the prementum - and retractile antennae, a unique feature among Chironomidae (Andersen et al., 2013). Their mandibles are armed with a very long apical tooth, and compared to more apomorphic subfamilies, they never build tubes but move freely on the substratum surface or in vegetation instead (Vallenduuk and Moller Pillot, 2007).

These characters correspond well with (at least occasional) predacious feeding by Tanypodinae larvae as a whole (Leathers, 1922; Baker and McLachlan, 1979), as confirmed in various species and generally accepted (review in Vallenduuk and Moller Pillot, 2007). Some evidence for tanypod predation consists of direct experimental observations (Leathers, 1922; Loden, 1974; Hershey, 1986), but mostly of findings of the remnants of the ingested prey in the gut (Hildrew et al., 1985; Armitage, 1968; Hershey 1986) along with larval mouthpart morphology (Gouin, 1959; Bryce and Hobart, 1972, cited in Baker and McLachlan, 1979). Some species have been reported to be unable to complete larval development if they could not feed upon animal prey (Vodopich and Cowel, 1984). However, not all individuals feed only on animal prey, and not for their whole larval lifespan (Armitage, 1968; Hildrew et al., 1985). When larvae are small they may be incapable of catching prey, and thus are believed to rely on detritus or algae (Baker and McLachlan, 1979). The same assumptions hold for small species, and also for periods of low prey density (e.g., in winter, Armitage, 1968; Hildrew et al., 1985). As the larvae grow 
bigger, the proportion of prey they consume increases (Baker and McLachlan, 1979; Hildrew et al., 1985).

Nevertheless, material of plant origin like detritus and algae is commonly found in the guts of Tanypodinae larvae, often in high amounts. There is a continuing debate about the origin of this food - it may be either directly consumed, or it may come from the intestines of prey which have just been sucked out (Leathers, 1922; Wesenberg-Lund 1943 cited in Armitage, 1968; Belyavskaya and Konstantinov, 1956 cited in Vallenduuk and Moller Pillot, 2007). Such ability of Tanypodinae to suck out their prey's inner tissues, together with body fluids and intestines, makes it impossible to determine their feeding strategy based solely on the gut content analysis. The gut contents may have come from the surrounding environment as well as from the intestines of a victim. Moreover, sucking out prey is more frequent in small individuals compared to larger ones (Belyavskaya and Konstantinov, 1956 cited in Vallenduuk and Moller Pillot, 2007), obviously because the small ones have too small mouth opening for the prey to pass.

Feeding mode and target resources are important features of organisms, which determine their functional position in ecosystems and also their ability to survive in a given environment. That is why feeding of aquatic macroinvertebrates is treated in all relevant autecological databases. However, there remain some discrepancies among the autecological databases and current literature regarding the feeding of Tanypodinae, partly because of the unreliability of gut content analysis, and partly because of the lack of thorough behavioural studies. The discrepancy is probably most apparent probably in the case of $M$. tenuicalcar.

European macroinvertebrate autecological databases (Moog, 2002; Tachet et al., 2010; Schmidt-Kloiber and Hering, 2015) generally treat representatives of the subfamily Tanypodinae as predators, with the least predacious genus being Procladius (with 6 out of 10 points assigned to predation and the remaining 4 to feeding on detritus; Schmidt-Kloiber and Hering, 2015) and with $M$. tenuicalcar classified as fully predaceous.

Unfortunately, the assignment of taxa to autecological categories in these databases is based mostly on experts' opinions (to be precise, Schmidt-Kloiber and Hering, 2015 used the classifications by Moog, 2002). Even when such expert opinion is sound, it is usually impossible to find the original source of information - reducing the appeal of such databases. Moreover, Tachet et al. (2010) treat Chironomidae only at the subfamily level, which is usually very imprecise and of little use for chironomidologists.

Recently, there has been an attempt by Serra et al. (2016) to compile all published autecological information on Chironomidae in a new database. This database is now being used in important ecological works (Serra et al., 2017a, 2017b), and according to it $M$. tenuicalcar is partly a shredder and partly a grazer/scraper - not a predator at all! This information was (imprecisely) taken from Vallenduuk and Moller Pillot (2007), who state that: "Detritus and green algae have been found in the gut, but the food of this species has been inadequately investigated. Most probably the larvae live on vegetable material and only incidentally some small animals."

The aim of this study was to shed light on the feeding behaviour of $M$. tenuicalcar, particularly to investigate whether this species does predate on larvae of other Chironomidae species co-occurring in the same environment. To do so, a laboratory experiment was carried out in which the larvae of $M$. tenuicalcar were offered larvae of Corynoneura sp. to make measurements and observations on the possible predacious activity of Monopelopia.

\section{METHODS}

All larvae used in the laboratory experiment were collected from the same peat pool $\left(49^{\circ} 39^{\prime} 24^{\prime \prime} \mathrm{N}\right.$, $15^{\circ} 53^{\prime} 14.5^{\prime \prime} \mathrm{E}$ ) on 23 March 2015. The pool is a part of a mineral poor Sphagnum fen, Radostínské rašeliniště National Nature Reserve in the Žd'árské Vrchy Protected Landscape Area, located within the Bohemian-Moravian Highlands. The water $\mathrm{pH}$ at the locality was around 3.54 during sampling. Due to the acid water the assemblage of chironomid larvae is simple with only several species, in early spring predominately $M$. tenuicalcar and Corynoneura sp., and later with a few species of Psectrocladius (Syrovátka and Langton, 2016). Worth noting is also the first record of the subfamily Podonominae for the Czech Republic within this same locality, represented by a stable population of Lasiodiamesa gracilis (Kieffer 1924) (Syrovátka and Langton, 2015). The vegetation at the site consists of the Sphagnion cuspidati association, with Sphagnum cuspidatum, Warnstorfia fluitans and Sphagnum phallax the dominant moss species. Eriophorum angustifolium, Oxycoccus palustris, Carex canescens and Carex rostrata are the most common vascular plants at the site.

To collect the larvae a hand net with mesh size of 237 $\mu \mathrm{m}$ was used to sweep submerged Sphagnum spp. moss within a small area (about $0.125 \mathrm{~m}^{2}$ ) to ensure that the larvae were collected from the same environment. Long Sphagnum spp. stems were gently rinsed and the flushed material was transferred into a plastic box. This material was stored in a fridge for two days before processing. On 25 March the larvae were carefully picked from the sample using a glass dropper in order not to cause injury and distributed in Petri dishes as follows: each of 32 Petri dishes received 8 larvae of Corynoneura sp. (prey) plus three Sphagnum stems 10-15 mm long, collected from the same 
sample, to serve as substratum. Then, $0,1,25$, or 4 larvae of $M$. tenuicalcar (predator) were added to establish four treatments, each with 8 replicates. The inner diameter of the Petri dishes used was $3.2 \mathrm{~cm}$, the area about $8 \mathrm{~cm}^{2}$. The Petri dishes were distributed randomly on a table at room temperature (around $22^{\circ} \mathrm{C}$ ) and checked under the dissecting microscope after 13.5, 19.5, 25, 35, 40, 58, and 84 hours to count living and dead larvae and their moulting.

The larvae of $M$. tenuicalcar used as predators in the experiment were most probably of the third instar, about $2.73 \mathrm{~mm}(2.40-2.84 \mathrm{~mm})$ long with the head length around $370 \mu \mathrm{m}$ (median $371 \mu \mathrm{m}$, range 313-397 $\mu \mathrm{m}$ ). Some of these moulted to the fourth instar during the experiment and reached body lengths up to $3.32 \mathrm{~mm}$ and head length up to $556 \mu \mathrm{m}$. None did pupate or die during the experiment.

Larvae of Corynoneura sp. are free living, and body lengths of larvae used ranged from 1.22-1.81 mm (median $1.52 \mathrm{~mm}$ ); their median head length was $200 \mu \mathrm{m}$ (range 161-245 $\mu \mathrm{m}$ ). As a considerable number of specimens was needed (256), both third and fourth instar Corynoneura were used as prey. Some of these larvae moulted to the fourth instar during the experiment, and some started to pupate after 40 hours. Therefore the experiment was terminated at that time. The observations on surviving prey larvae continued for two more days and stopped after 84 hours since the start, when most individuals of Corynoneura sp. emerged as adults or died. All the emerged individuals were females and two of them laid eggs in the Petri dish, demonstrating their ability to reproduce parthenogenetically. For a visual comparison of the sizes of the larvae of both species, see Fig. 1.

As the mortality of Corynoneura sp. larvae in the

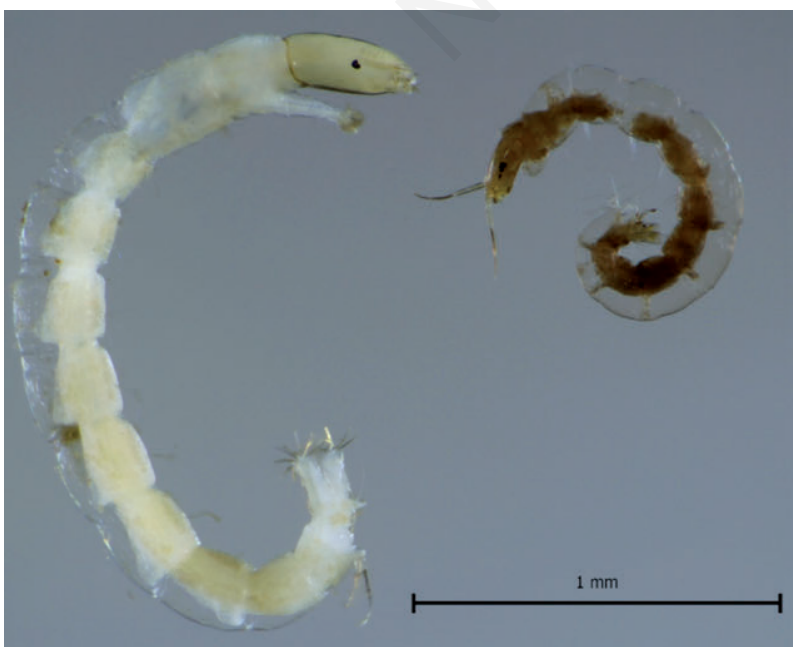

Fig. 1. An example of Monopelopia tenuicalcar (left) and Corynoneura sp. (right) larvae used in the experiment. control group was not zero (two specimens died during the 40-hour experiment), the predatory effect of $M$. tenuicalcar larvae was estimated as the difference between the "natural" mortality observed in the control group and the mortality observed in the other treatments. For simplicity, the predatory effect was modelled as the proportion of larvae that died during the first 40 hours of the experiment compared to the control treatment via binomial Generalized Linear Models. Three comparisons were made in order to estimate and compare also the predatory pressure of different $M$. densities. The following hypotheses were tested:

i. There was no difference in the mortality of Corynoneura sp. larvae between the control group and the group with one larva of $M$. tenuicalcar.

ii. There was no difference in the mortality of Corynoneura sp. larvae between the treatment groups with one and two larvae of M. tenuicalcar.

iii. There was no difference in the mortality of Corynoneura sp. larvae between the treatment groups with two and four larvae of $M$. tenuicalcar.

Data were analysed and visualised in $\mathrm{R}$ version 3.4.1 (R Core Team, 2017), and comparisons among treatments were tested using the package multcomp (Hothorn et al., 2008).

\section{RESULTS}

\section{Monopelopia predation effect on Corynoneura larval mortality}

After 40 hours since the start of the experiment, there was a notable effect of $M$. tenuicalcar larvae on the mortality of Corynoneura sp. larvae (Fig. 2). The average mortality in the control group was 0.25 larvae per Petri dish (which corresponds to 2 dead larvae out of the 64 larvae in 8 dishes). In treatments with $M$. tenuicalcar larvae, mortality was an order of magnitude higher: 2.375 , 2.875, and 5.5 larvae per Petri dish on average in the presence of 1,2 , and $4 M$. tenuicalcar larvae, respectively. The statistical comparisons revealed that the mortality of Corynoneura sp. larvae was significantly higher in the presence of $1 \mathrm{M}$. tenuicalcar compared to the control group $(\mathrm{P}=0.0025)$, suggesting a strong predatory effect of M. tenuicalcar larvae. The presence of two Monopelopia larvae did not cause a significantly higher mortality of Corynoneura as compared to the mortality caused by one M. tenuicalcar $(\mathrm{P}=0.8159)$ but four $M$. tenuicalcar larvae exhibited a significantly higher predatory pressure than two predators per dish $(\mathrm{P}<0.001)$.

\section{Behavioural observations}

While attempting to record a video of the attack of $M$. tenuicalcar, the author has observed many cases when $M$. 
tenuicalcar met with Corynoneura. Corynoneura always was the more active element crawling freely around, with its antennae extended far in front of its head and grazing on algae and biofilm growing on the surface of moss or the Petri dish. M. tenuicalcar moved much more slowly with long breaks, during which it was just turning its head around with antennae protruding back and forth. If Corynoneura approached $M$. tenuicalcar from the front, once their antennae touched Corynoneura immediately withdrew by a backward flexing of the posterior end of the body. In such situations the attempts by M. tenuicalcar to attack Corynoneura were unsuccessful. The author has only observed successful attacks when Corynoneura passed around $M$. tenuicalcar, which could then attack Corynoneura from the side. In such case Corynoneura did not expect the attack and also the usual escape backwards would not help much.

After the attack, once Corynoneura was pierced by the mandibles of $M$. tenuicalcar, there was a short fight during which Corynoneura tried to escape by wincing and twisting. This is probably the last chance for the prey to escape. After M. tenuicalcar started to suck the body fluid of Corynoneura, Corynoneura almost stopped moving and waited calmly for death. Sucking is performed with the use of head muscles and the ligula, which is repeatedly reflexed from the ventral-forward position through a dorsal position and backwards, creating a strong suction pressure and scraping against the body wall of the victim.

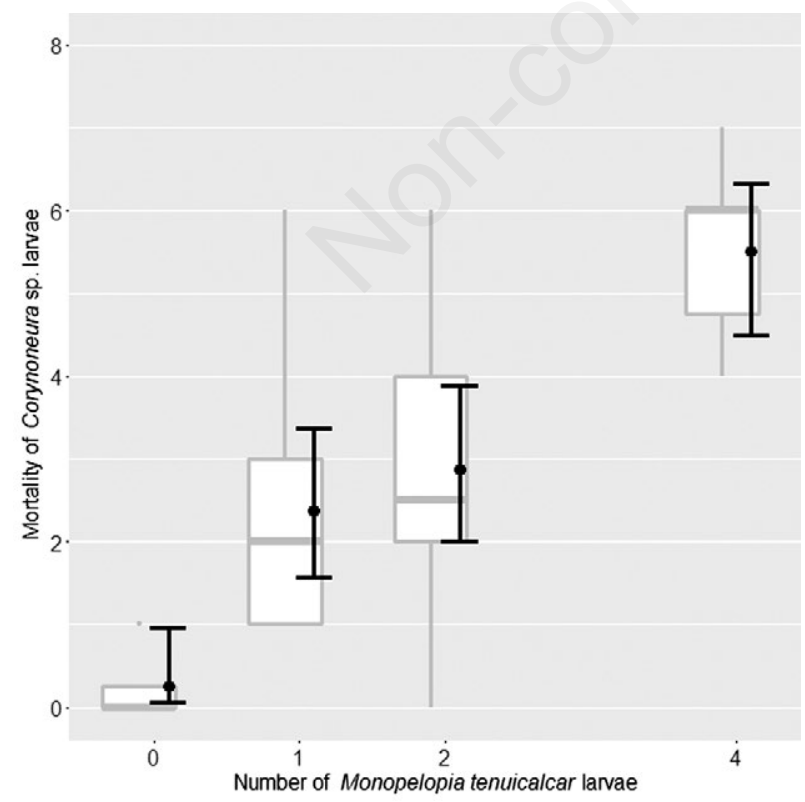

Fig. 2. The observed (boxplot) and modelled (black dot, mean; black whiskers, 95\% confidence intervals) mortality of Corynoneura sp. larvae under various densities of Monopelopia tenuicalcar larvae after 40-hour exposition.
The sucked fluid is partly visible through the translucent body wall of $M$. tenuicalcar; in some cases it seemed to me that $M$. tenuicalcar also regurgitated its gut content back into the body of the victim, so it is possible that digestion may take place partly inside the body of the prey. Several larvae of $M$. tenuicalcar may suck one prey at a time but the author observed this only in high densities of $M$. tenuicalcar larvae and after they were starved (not during the experiment).

During the experiment, 86 Corynoneura larvae died in the experimental groups with $M$. tenuicalcar larva/larvae. Out of them, only two were missing completely, and were presumed to have been engulfed. The remaining 84 prey carcases had been just sucked out. The author also observed a single case of complete ingurgitation. The video of this event is available at https://youtu.be/o6-8dA1XDy0.

During the 2015 experiments reported above, the author never observed $M$. tenuicalcar larva ingesting any vegetable matter. Subsequently in 2017, the author recorded a larva as it clearly and intentionally sucked a fluff of algae lying on the bottom of the Petri dish. That larva was, however, from a different locality: a small, well-vegetated pool much richer in nutrients (not a Sphagnum bog) and most probably also with a different algal community.

Interestingly, the author has personally never observed cannibalism in $M$. tenuicalcar larvae, and also no $M$. tenuicalcar died during the experiment. However, when the larvae were kept at high density (about one hundred larvae in one Petri dish), some of them were sucked out after several days. Whenever two $M$. tenuicalcar larvae met, they always turned to face each other head-to-head and began mutual touching with antennae and mandibles. They also appeared to attempt to bite each other, but always met only their opponent's mandibles. It is possible that at high densities a larva could not defend itself if attacked by more than one larva (i.e. from multiple sides), but that circumstance may rarely occur in nature.

\section{DISCUSSION}

This study brings evidence of predation by $M$. tenuicalcar on another chironomid larvae. This result agrees with Schmidt-Kloiber and Hering (2015) and contradicts Serra et al. (2016). The behaviour of $M$. tenuicalcar while hunting - slow movements with frequent pauses, and turning of the head as if sensing the immediate environment - resembles a careful search for prey observed in other Tanypodinae (Leathers, 1922; Baker and McLachlan, 1979) and is in stark contrast to the restless grazing or collecting by Corynoneura spp. or other chironomids such as some Psectrocladius spp., Spempellina bausei (Kieffer, 1911), and Neostempellina 
thienemanni Reiss, 1984, which the author also has observed, and which are not limited in their movements by tubes attached to the substratum. In this respect the author cannot agree with Fittkau (1962), who states about M. tenuicalcar that "Die Larven erinnern in Gestalt und Bewegung an Larven von Corynoneura" [The larvae (of Monopelopia) resemble those of Corynoneura in their form and movement].

In agreement with Leathers (1922) the author believes that similar morphology and behaviour within Tanypodinae predispose all Tanypodinae to feed primarily as predators (as they are already classified in Moog, 2002 and later in Schmidt-Kloiber and Hering, 2015), and when lacking prey, they utilize also alternative resources like detritus and algae (Morgan, 1949). Results reported here support this view. Plant-based food does not seem to be the primary source of energy for M. tenuicalcar however, even if algae and detritus may represent all the recognizable contents of the gut. Piercing the prey and sucking out its contents is probably more frequent than has been thought. Although this feeding strategy is well-known within the Tanypodinae (Leathers, 1922; Vallenduuk and Moller Pillot, 2007), the author knows of no other study to quantify the proportion of prey pierced and sucked, versus being totally ingested. In this study, piercing and sucking comprised $98 \%$ of the predation events, meaning the vast majority of prey would probably not have been identified in the gut of these predaceous $M$. tenuicalcar larvae by conventional microscopic methods. Tanypodinae larva may use the piercing/sucking predation mode whenever their prey is small enough to capture, but its rigid, chitinous structures are too large to fit the predator's mouth opening (Leathers, 1922; Belyavskaya and Konstantinov, 1956 as cited in Vallenduuk and Moller Pillot, 2007). Regardless of whether the prey is ultimately sucked out or engulfed whole, the predator's procedure for seizing and processing it is the same, as described by Leathers (1922) for "Tanypus carneus" [most probably Thienemannimyia carnea (Fabricius, 1805)]. Mandibles are employed to hold the prey and a powerful sucking apparatus composed mostly of ligula and strong muscles, is used to suck the prey. If the prey fits the mouth opening of the predator, it is engulfed completely, otherwise it is just sucked out.

Taking into account that the sizes of larvae used in the current study reflected the natural size distributions of both predators and prey at the time of the experiment, it is probable that similar prey-consumption patterns occur anywhere in nature, and that piercing and sucking out prey could dominate feeding in Tanypodinae generally. On the other hand, as the present study involved only one tanypod species, one must be cautious about drawing a general conclusion regarding all Tanypodinae larvae. In any case, the possibility that sucking out of prey is a widespread feeding strategy within the Tanypodinae emphasizes the uncertainty of gut content analysis for determining the larval feeding mode in this chironomid subfamily.

Corynoneura spp. larvae are free-living without tubes or cases, and it may be more difficult for Tanypodinae larvae to detect and feed on larvae that live in tubes. On the other hand, Corynoneura larvae possess other adaptations to avoid predators, including long, sensitive antennae. Moreover, some Tanypodinae have been reported to use tubes of chironomids to assist in prey capture (Izvekova, 1980 cited in Vallenduuk and Moller Pillot, 2007). While Hershey (1987) showed tube dwelling to influence the susceptibility of chironomid larvae to a damselfly predator, comparative analyses of the vulnerability of tube-building, versus free-living, chironomid larvae to tanypod predation are needed.

Interestingly, two larvae of $M$. tenuicalcar did not cause higher mortality of Corynoneura sp. than just one predatory larva. Such interference among predators is well known in other systems (Beddington, 1975). In this experiment, another doubling of the predator density (from 2 larvae to 4 larvae) then outweighed the initial predator interference and resulted in higher overall prey consumption.

In the light of the results of this study, the classification by Serra et al. (2016) of M. tenuicalcar as partly a shredder and partly a grazer/scraper seems incorrect. At the least, predation should be included in the feeding strategy of this species. The database by Serra $e t$ al. (2016) also disagrees with Schmidt-Kloiber and Hering (2015) regarding the feeding of Tanypodinae. Serra et al. (2016) assign "shredding" (as well as "grazing/scraping") to Apsectrotanypus trifascipennis (Zetterstedt, 1838), M. tenuicalcar, and the genera Ablabesmyia, Arctopelopia, Conchapelopia, Procladius, Trissopelopia, and Zavrelimyia. They also assign "filter feeding" to Ablabesmyia, Tanypus (Tanypus), Telmatopelopia, Thienemannimyia, Trissopelopia, Xenopelopia, and Zavrelimyia. None of these feeding strategies is assigned to any representative of Tanypodinae in Schmidt-Kloiber and Hering (2015). Most of these feeding modes listed for Tanypodinae larvae by Serra et al. (2016), are attributed to publications by Vallenduuk and Moller Pillot (2007) and Hildrew et al. (1985). Those cited authors, however, do not mention shredding or filter feeding by any Tanypodinae. The occurrence of detritus or organic matter in the gut, reported by Vallenduuk and Moller Pillot (2007), may have been misinterpreted by Serra et al. (2016). The criteria used by Serra et al. (2016) for assignment of feeding strategies to taxa in their database is unclear. For example, Arctopelopia and Thienemannimyia both feed on animals, algae and detritus according to Vallenduuk and Moller Pillot (2007) - the sole reference for these two genera, but Serra et al. (2016) assign very different feeding strategies to these two 
genera, listing "predator, shredder and grazer/scraper for Arctopelopia, and "predator, fine sediment feeder, and filter feeder" for Thienemannimyia. Moreover, Macropelopia and Psectrotanypus varius are treated as fully predacious by Serra et al. (2016), while Vallenduuk and Moller Pillot (2007) report also vegetarian food for these taxa.

Despite these apparent errors, the idea of establishing a well-founded database derived from published evidence is commendable. However, the foundation of any such classification must include clear criteria and traceable sources if it is to become a reliable resource.

Future research ought to focus on detailed behavioural and experimental studies of various Tanypodinae species, accompanied with stable isotope analysis (Peterson and Fry, 1987) to ascertain the trophic position of species within aquatic ecosystems. Such new evidence would also enable the revision of previous findings that have been based on (potentially unreliable) gut content analysis. Information in what may be the most comprehensive and up-to-date database maintained by Schmidt-Kloiber and Hering (2015), www.freshwaterecology.info, is recommended. This resource includes valuable information on Chironomidae as well as all aspects of aquatic biology, based on multiple contributors and long periods of cumulative experience.

\section{CONCLUSIONS}

The larvae of $M$. tenuicalcar demonstrated a clear predatory behaviour, but on one occasion a larva was also observed to ingest algae. Direct observations of feeding by Tanypodinae larvae are, however, still very rare. Piercing and sucking out prey by Tanypodinae is probably more common than has been appreciated. For this reason, gut content analysis seems to be unreliable for the determination of feeding strategy in Tanypodinae, and the assignment of feeding strategies derived from gut content is not recommended.

\section{ACKNOWLEDGMENTS}

I am deeply grateful to reviewers Malcolm G. Butler and Henk J. Vallenduuk for helpful comments and significant improvements to the manuscript. This study was supported by the Czech Science Foundation (Project no. P505/16-03881S).

\section{REFERENCES}

Andersen T, Cranston PS, Epler JH, 2013. Chironomidae of the Holarctic Region: Keys and diagnoses - Larvae. Entomological Society, Lund: 571 pp.
Armitage PD, 1968. Some notes on the food of the chironomid larvae of a shallow woodland lake in South Finland. Ann. Zool. Fenn. 5:6-13.

Ashe P, O'Connor JP, 2009. A World catalogue of Chironomidae (Diptera). Part 1. Buchonomyiinae, Chilenomyiinae, Podonominae, Aphroteniinae, Tanypodinae, Usambaromyiinae, Diamesinae, Prodiamesinae and Telmatogetoninae. Irish Biogeographical Society and National Museum of Ireland, Dublin: 445 pp.

Baker AS, McLachlan AJ, 1979. Food preferences of Tanypodine larvae (Dptera: Chironomidae). Hydrobiologia 62:283-288.

Beddington JR, 1975. Mutual interference between parasites or predators and its effect on searching efficiency. J Animal. Ecol. 44: 31-340.

Belyavskaya LI, Konstantinov AS, 1956. [The feeding of larvae Procladius choreus Meig. (Chironomidae, Diptera) and the loss they cause to the food base of fishes].[Article in Russian]. Vopr. Ikhtiol. 7:193-203.

Bryce D, Hobart A, 1972. The biology and identification of the larvae of the Chironomidae (Diptera). Ent. Gaz. 23:175-217.

Fittkau EJ, 1962. [Die Tanypodinae (Diptera, Chironomidae). Die Tribus Anatopyniini, Macropeloplini und Pentaneurini]. [Article in German]. Abh. Larvalsyst. Insekten 6:1-453.

Gouin FJ, 1959. Morphology of the larval head of some Chironomidae (Diptera, Nematocera). Smithsonian Miscellaneous Collections 137:175-201.

Hershey AE, 1986. Selective predation by Procladius in an arctic Alaskan lake. Can. J. Fish. Aquat. Sci. 43:2523-2528.

Hershey AE, 1987. Tubes and foraging behavior in larval Chironomidae: implications for predator avoidance Oecologia 73:236-241.

Hildrew AG, Townsend CR, Hasham A, 1985. The predatory Chironomidae of an iron-rich stream: feeding ecology and food web structure. Ecol. Entomol. 10:403-413.

Hothorn T, Bretz F, Westfall P, 2008. Simultaneous inference in general parametric models. Biometrical J. 50:346-363.

Izvekova EI, 1980. [The food of chironomids]. In: Bentos Uchinskogo vodokhranilishcha. [Article in Russian]. Trudy vses. gidrobiol. Obshch. 23:72-101. Izd. Nauka, Moskva.

Leathers AL, 1922. Ecological study of aquatic midges and some related insects with special reference to feeding habits. Bull. U. S. Bureau Fish. 38:1-61.

Loden MS, 1974. Predation by Chironomid (Diptera) larvae on Oligochaetes. Limnol. Oceanogr. 19:156-59.

Moog O, 2002. Fauna Aquatica Austriaca. Wasserwirtschafskataster, Bundesministerium für Land- und Forstwirtschaft, Umwelt und Wasserwirtschaft, Vienna.

Morgan MJ, 1949. The metamorphosis and ecology of some species of Tanypodinae (Dipt., Chironomidae). Entomol. Mon. Mag. 85:119-126.

Peterson BJ, Fry B, 1987. Stable isotopes in ecosystem studies. Annu. Rev. Ecol. Syst. 18:293-320.

R Core Team, 2017. R: A language and environment for statistical computing. $\mathrm{R}$ Foundation for Statistical Computing, Vienna, Austria. Available from: https://www.Rproject.org/

Schmidt-Kloiber A, Hering D, 2015. www.freshwaterecology.info - an online tool that unifies, standardises and codifies more than 20,000 European freshwater organisms 
and their ecological preferences. Ecol. Indic. 53:271-282.

Serra SRQ, Cobo F, Graca MAS, Dolédec S, Feio MJ, 2016.

Synthesising the trait information of European Chironomidae (Insecta: Diptera): Towards a new database. Ecol. Indic. 61:282-292.

Serra SRQ, Graça MAS, Dolédec S, Feio MJ, 2017a. Chironomidae traits and life history strategies as indicators of anthropogenic disturbance. Environ. Monit. Assess. 189:326.

Serra SRQ, Graça MAS, Dolédec S, Feio MJ, $2017 \mathrm{~b}$. Chironomidae of the Holarctic region: a comparison of ecological and functional traits between North America and Europe. Hydrobiologia 794:273-285.

Syrovátka V, Langton PH, 2015. First records of Lasiodiamesa gracilis (Kieffer, 1924), Parochlus kiefferi (Garrett, 1925) and several other Chironomidae from the Czech Republic and Slovakia. CHIRONOMUS J. Chironomidae Res. 28:45-56.

Tachet H, Bournaud M, Richoux P, Usseglio-Polatera P, 2010. [Invertébrés d'eau douce - systématique, biologie, écologie].[Book in French]. CNRS Editions, Paris: 600 pp.

Vallenduuk HJ, Moller Pillot HKM, 2007. Chironomidae larvae. General ecology and Tanypodinae. KNNV Publishing, Zeist: 144 pp.

Vodopich DS, Cowell BC, 1984. Interaction of factors governing the distribution of a predatory aquatic insect. Ecology 65:39-52.

Wesenberg-Lund C, 1943. [Bemerkungen über die Biologie der Chironomiden].[Article in German]. Entomol. Medd. 23:179-203. 